\title{
Frequência de excesso de peso em pré-escolares
} Overweight frequence in preschool

\author{
William Cordeiro de Souza', Valderi Abreu de Lima², Luis Paulo Gomes Mascarenhas \\ 'Universidade Estadual do Centro-Oeste (Unicentro), Irati, PR, Brasil. \\ ¿Universidade Federa do Paraná (UFPR), Curitiba, PR, Brasil.
}

Recebido em: 31/03/2015 / Aceito em: 16/04/2016

professor_williamsouza@yahoo.com.br

\section{RESUMO}

Objetivo: avaliar a frequência de excesso de peso em pré-escolares. Método: a amostra foi constituída por 151 pré-escolares, sendo 71 do sexo masculino, com idade média de $5 \pm 0,1$ anos. Para avaliação do estado nutricional, foi avaliado o peso corporal e a estatura, para obtenção do índice de massa corporal (IMC). Na classificação do IMC, utilizou-se como referência as curvas de percentis, recomendado pelo Center for Disease Control and Prevention. Para análise dos dados foi utilizada a estatística descritiva: média, desvio padrão, frequência percentual e para verificar as diferenças entre os sexos foi utilizado o teste $t$ de Student para amostras independentes. Recorreu-se ao teste qui-quadrado para verificar as diferenças entre as frequências percentuais. Foi adotado um nível de significância estimado em $\mathrm{p}<0,05$. Resultados: no geral, a frequência de sobrepeso foi de $19,20 \%$ e de obesidade foi de $9,93 \%$, respectivamente. Entre os meninos, a frequência de sobrepeso foi $29,57 \%$ e de obesidade de $5,63 \%$. Já, nas meninas, a frequência de sobrepeso foi de 10,00\% e $13,75 \%$ de obesidade. Considerações finais: foi verificada uma alta frequência de sobrepeso e obesidade na população estudada, cabe ressaltar que as proporções obtidas de excesso de peso não resultaram em valores significativos quando comparado às classificações do grupo meninos em relação às meninas. tricional.

Palavras-chave: Obesidade; Crianças; Estado Nu-

\section{ABSTRACT}

Objective: to assess the frequency of overweight in preschool. Method: the sample was composed by 151 preschool children, 71 male and 80 female, with a mean age of $5 \pm 0.1$ years. To evaluate the nutritional status was assessed body weight and height to obtain the body mass index (BMI). The BMI classification was used as the reference percentile curves recommended by the Centers for Disease Control and Prevention. Data analysis was used descriptive statistics: mean, standard deviation, frequency and percentage to check the differences between genders, we used the Student $t$ test for independent samples. It used the chi-square test to check the differences between the percentage frequencies. A significance level estimated at $p<0.05$ was adopted. Results: Overall, the frequency, the prevalence of overweight was $19.20 \%$ and obesity was 9.93\%, respectively. The overweight rate was $29.57 \%$ and $5.63 \%$ obesity among boys. Already, in girls the overweight rate was $10.00 \%$ and $13.75 \%$ of obesity. Closing remarks: a high frequency of overweight and obesity in the population studied was found, it should be noted that the obtained overweight proportions did not result in significant amounts when compared to boys group rankings over girls.

Keywords: Obesity; Children; Nutritional Status.

\section{INTRODUÇÃO}

Altos índices de sobrepeso e obesidade têm aumentado de forma significativa em todo mundo e essa epidemia está sendo considerada um problema de saúde pública. ${ }^{1}$ Cabe destacar que o crescimento desenfreado da obesidade vem crescendo, tanto nos países desenvolvidos, como nos subdesenvolvidos. ${ }^{2}$

No Brasil, nas últimas décadas essa epidemia apresentou aumento considerável em todas as faixas etárias, mas em especial, destaca-se a população infantil e adolescente. ${ }^{3}$ Segundo dados da Pesquisa e Orçamentos Familiares de 2008 e 2009, estimam que $33 \%$ das crianças brasileiras entre 5 a 9 anos de idade estejam acima do peso, sendo que, $14,3 \%$ já são con- 
sideradas obesas. ${ }^{4}$

O Instituto Brasileiro de Geografia e Estatística $\left(\right.$ IBGE) ${ }^{5}$ destaca que desde a década de 1970, triplicou o número de escolares brasileiros com sobrepeso e, nos dias atuais, existem mais adolescentes acima do peso do que desnutridos. Segundo informações do Instituto Nacional de Alimentação e Nutrição, ${ }^{6}$ isto ocorreu devido modificações no estilo de vida da população, onde reduziu a desnutrição e houve um aumento progressivo da obesidade infantil, caracterizando um processo de transição nutricional.

Vale ressaltar que o estado nutricional desejável de uma criança possui papel fundamental para que seu crescimento seja progressivo e para que ela desenvolva suas aptidões psicomotoras e sociais. Alterações de déficit ou excesso expõem tais crianças a riscos potenciais de agravos à saúde, e problemas futuros de relações interpessoais e funcionais dentro da comunidade. ${ }^{7}$

O desenvolvimento do sobrepeso e da obesidade tem sido constatado em idades cada vez mais precoces, especialmente em pré-escolares. Este fenômeno, nesta fase, demonstra associação com a obesidade na idade adulta e consequentemente está relacionado com as doenças crônicas associadas. ${ }^{8}$

Assim, diante da importância de estudar a saúde da criança e a obesidade, que tem se manifestado cada vez mais precocemente e com consequências graves, este estudo é importante para avaliar o estado nutricional de crianças pré-escolares e fornecer dados aos profissionais de saúde para melhor atuar nas medidas de proteção à saúde e prevenção dessa doença. Destarte, o presente estudo tem por objetivo avaliar a frequência de excesso de peso em pré-escolares.

\section{MÉTODO}

\section{Amostra}

A amostra intencional foi constituída por 151 pré-escolares matriculados em um Centro de Educação Infantil do município de Três Barras - SC, sendo 71 do sexo masculino com idades de 4 e 5 anos. Este estudo foi aprovado pelo Comitê de Ética em Pesquisa em Seres Humanos da Universidade do Contestado - UnC (parecer consubstanciado - CAAE: 05020512.7.0000.0117), estando de acordo com a resolução $n^{\circ}$ 466/2012 do Conselho Nacional de Saúde. Todos os participantes foram informados dos objetivos do projeto, concordaram e assinaram o Termo de Consentimento Livre e Esclarecido (TCLE).

\section{Critérios de Inclusão e Exclusão}

As crianças incluídas no estudo deveriam estar regularmente matriculadas na escola selecionada para a pesquisa, ter idade entre 4 e 5 anos, tomando como base o mês de março de 2016, apresentar o TCLE as- sinado pelos pais ou responsáveis. Foram excluídas do estudo, crianças que apresentaram doenças crônicas ou específicas do crescimento, que não compareceram na escola nos dias marcados para coleta de dados e as crianças cujos pais não autorizaram a participação, sendo também respeitada a vontade das crianças que se recusaram a participar mesmo com a autorização dos pais.

\section{Avaliação Antropométrica}

Para avaliação do estado nutricional, foi avaliado o peso corporal e a estatura para obtenção do índice de massa corporal (IMC), utilizando-se à seguinte formula: IMC $=$ Peso Corporal/ Estatura. ${ }^{2} \mathrm{Na}$ mensuração do peso corporal, o avaliado deveria se posicionar em pé, de costas para escala da balança, usando o mínimo de roupa possível. ${ }^{9}$ Foi utilizada uma balança digital da marca Techline (fabricada no Brasil), devidamente calibrada, com graduação de 100 gramas e escalas, variando de 0 a $180 \mathrm{Kg}$.

A estatura foi identificada pelo maior valor entre o vértex e a região plantar, obedecendo ao plano de Frankfurt. ${ }^{9} \mathrm{~A}$ estatura foi verificada através de uma trena flexível marca Sanny Medical Sparrett, (fabricada no Brasil) resolução de 0,1 $\mathrm{mm}$, fixada na parede lisa, com 3 metros e graduação de $0,1 \mathrm{~cm}$ com o zero coincidindo com o solo.

Para a classificação do IMC, utilizou-se como referência as curvas de percentis, recomendado pelo Center for Disease Control and Prevention, ${ }^{10}$ que classifica como baixo IMC para idade valores < percentil 5, IMC adequado ou eutrófico $>$ percentil 5 e $<$ percentil 85, sobrepeso $>$ percentil 85 e $<$ percentil 95 e para obesidade valores $>$ percentil 95. Através desses dados, foi classificado o sobrepeso e obesidade nas crianças.

\section{Análise dos Dados}

$\mathrm{Na}$ análise dos dados foi utilizado à estatística descritiva: média, desvio padrão (dp), frequência percentual (\%) e para verificar as diferenças entre os sexos, foi utilizado o teste $t$ de Student para amostras independentes. Recorreu-se ao teste qui-quadrado para verificar as diferenças entre as frequências percentuais das classificações do estado nutricional. Foi adotado um nível de significância estimado em $\mathrm{p}<0,05$. Os dados foram analisados através do software BioEstat 5.0.

\section{RESULTADOS}

A tabela 1 apresenta a comparação dos dados antropométricos para caracterização da amostra. É possível observar que não foram encontradas diferenças significativas em ambos os sexos.

A tabela 2 apresenta os percentuais das classificações obtidas no IMC. É possível observar uma alta frequência de sobrepeso e obesidade na população estudada, sendo maior do sobrepeso nos meninos.

Tabela 1 - Caracterização da amostra.

\begin{tabular}{|c|c|c|c|c|}
\hline Variáveis & $\begin{array}{c}\text { Meninos } \\
(\mathrm{n}=71)\end{array}$ & $\begin{array}{c}\text { Meninas } \\
(\mathrm{n}=80)\end{array}$ & $\mathbf{T}$ & $\mathbf{p}$ \\
\hline Massa Corporal (kg) & $20,0 \pm 3,51$ & $20,0 \pm 4,65$ & $-0,291$ & 0,385 \\
\hline Estatura (m) & $1,10 \pm 0,6$ & $1,10 \pm 0,6$ & $-0,061$ & 0,475 \\
\hline IMC (kg/m²) & $16,01 \pm 1,58$ & $16,14 \pm 2,57$ & $-0,358$ & 0,360 \\
\hline
\end{tabular}

$\mathrm{n}=$ número de indivíduos por grupo. 
Tabela 2 - Caracterização da amostra.

\begin{tabular}{lcc}
\hline & \multicolumn{2}{c}{ Meninos $(\mathbf{n}=\mathbf{7 1})$} \\
\hline IMC & $\mathbf{n}$ & $\%$ \\
Eutrófico & 46 & 64,78 \\
Sobrepeso/Obesidade & 25 & 35,22 \\
& Meninas $(\mathbf{n}=\mathbf{8 0})$ \\
\hline IMC & $\mathbf{n}$ & $\%$ \\
Eutrófico & 61 & 76,25 \\
Sobrepeso/Obesidade & 19 & 23,75 \\
& Total $(\mathbf{n}=151)$ \\
\hline IMC & $\mathbf{n}$ & $\%$ \\
Eutrófico & 107 & 70,86 \\
Sobrepeso/Obesidade & 44 & 29,14 \\
\hline Teste de qui-quadrado; $\mathbf{p}=0,122$ & \multicolumn{2}{c}{}
\end{tabular}

Quando comparada a classificação entre os sexos, não foram encontradas diferenças significativas.

\section{DISCUSSÃO}

O presente estudo com o intuito de avaliar a frequência de excesso de peso em pré-escolares, verificou que no geral, a frequência, a prevalência de sobrepeso foi de $19,20 \%$ e de obesidade foi de $9,93 \%$, respectivamente. A frequência de sobrepeso foi $29,57 \%$ e de obesidade de $5,63 \%$ entre os meninos. Já, nas meninas, a frequência de sobrepeso foi de 10,00\% e 13,75\% de obesidade. Nas classificações obtidas no IMC não foram encontradas diferenças significativas em ambos os grupos e no geral.

Em estudo realizado por Souza et al., ${ }^{11}$ os autores verificaram que $26,67 \%$ dos meninos avaliados apresentaram do peso acima do desejável e entre as meninas, $45,16 \%$. Dados esses que corroboram com o presente estudo, pois as maiores proporções de obesidade foram entre as meninas que nos meninos $(13,75 \%$ e 5,63\%, respectivamente). No estudo de Hoehr et al., ${ }^{12}$ a frequência de sobrepeso e obesidade avaliando 726 escolares do município de Santa Cruz do Sul - RS foi de 26,6 \%. Quando comparadas as classificações entre os sexos não houve diferença, sendo $26,59 \%$ para as meninas e $26,57 \%$ para os meninos. Resultado que corrobora com presente estudo.

Outro estudo desenvolvido por Souza et al..$^{13} \mathrm{com}$ objetivo de realizar um comparativo entre o sobrepeso e obesidade entre crianças pré-escolares de ambos os sexos, não foram encontradas diferenças significativas nas variáveis de idade, estatura e IMC, mas encontraram diferença no peso corporal dos meninos avaliados. As proporções demonstraram que o gênero masculino apresentou 30,35\% de sobrepeso/obesidade e as meninas $23,9 \%$. Resultados semelhantes foram encontrados no presente estudo. O estudo supracitado limitou-se em não realizar as comparações das proporções obtidas.

Panazollo et al. ${ }^{4}$ determinaram a prevalência de sobrepeso e obesidade entre escolares do ensino fundamental e verificaram que a prevalência de obesidade, no grupo avaliado, foi de $15,9 \%$ e o sobrepeso acometeu $15,4 \%$ das crianças. Quando somandas as duas condições, $31,4 \%$ dos escolares estavam acima do peso esperado para a idade e estatura. A prevalência de obesidade foi maior nas faixas etárias de 6 e 7 anos, quando comparada às das crianças de 9 e 10 anos, $23,3 \%$ e $8,23 \%$, respectivamente $(p<0,001)$. Meninos e meninas não apresentaram diferença significativamente estatística na prevalência de sobrepeso $(15,4 \%$ para $15,2 \%)$ e obesidade (16,1\% para 15,2\%). Em Pelotas-RS, um estudo demonstrou que o índice de obesidade infantil aumentou de $4 \%$ para $6,7 \%$, em um período de 11 anos. ${ }^{14}$

$\mathrm{O}$ conhecimento do estado nutricional e frequência de sobrepeso e obesidade em crianças são importantes, pois as consequências da obesidade infantil podem ser notadas a curto e longo prazo, que podem ir desde desordens ortopédicas, diabetes, hipertensão arterial, dislipidemias, problemas respiratórios até aumento da mortalidade por causas diversas, em geral associadas a doenças coronarianas, em adulto que foram obesos na infância. ${ }^{15}$

Em uma pesquisa realizada na cidade de Santos-SP, avaliando 10.822 crianças com idades entre 7 a 10 anos, encontrou-se um percentual de $18 \%$ de obesidade entre os avaliados. ${ }^{16} \mathrm{Em}$ estudo semelhante, realizado na cidade de Londrina-PR, a frequência de obesidade foi de $14 \% .{ }^{17}$

A frequência de sobrepeso e obesidade normalmente está associada a dois fatores, alimentação inadequada, geralmente com excesso de carboidratos na dieta e diminuição dos níveis de atividade física. ${ }^{18}$ Estas variáveis não foram avaliadas no presente estudo, porém é de suma importância que novos estudos sejam realizados com avaliação destes elementos para possíveis revisões nas políticas públicas de prevenção a saúde infantil, pois o combate da obesidade na infância pode evitar a obesidade na fase adulta. ${ }^{19}$

O presente estudo tem limitações e representa apenas um diagnóstico e parcial retrato da situação do estado nutricional de crianças pré-escolares. Outros parâmetros não foram analisados neste estudo como condições socioeconômicas, prática de atividade física e alimentação, variáveis que possuem relação direta com o estado nutricional.

É necessário que os governantes e os profissionais envolvidos na área da saúde e educação se envolvam mais nessa problemática, atentando para a importância de um estilo de vida mais saudável e implantação de ações de prevenção, promoção e tratamento da obesidade na infância e adolescência.

\section{CONSIDERACÕES FINAIS}

O presente estudo verificou uma alta frequência de sobrepeso e obesidade na população estudada. Cabe ressaltar que as proporções obtidas de excesso de peso não resultaram em valores significativos, quando comparado às classificações do grupo meninos em relação às meninas.

\section{REFERÊNCIAS}

1. Frignani RR, Passos MAZ, Ferrari GLM, Niskier SR, Fisberg M, Cintra IP. Reference curves of the body fat index in adolescents and their association with anthropometric variables. J Pediatr 2015;91(3);248-55. DOI: 10.1016/j. jped.2014.07.009. 
2. Lacerda RC, Melo FAT. Prevalência de sobrepeso e obesidade em escolares de uma instituição de ensino pública. Educação Física em Revista 2011;5(2):1-11.

3. Souza WC, Grzelzak MT, Reiser FC, Lima VA, Souza, WB, Alarcón-Meza El, et al. Relação entre o IMC e o IAC em meninos pré-escolares. Rev Bras Qualidade Vida 2015;7(1):48-55. DOI: 10.3895/rbqv.v7n1.2794.

4. Panazzolo PR, Finimundi HC, Stoffel MOS, Simon RA, Lima $\mathrm{MC}$, Costanzi CB. Prevalence of overweight and obesity in schoolchildren in the municipality of Feliz, Rio Grande do Sul state, Brazil. Rev Bras Med Fam Comunidade 2014;9(31):141 8. DOI: $10.5712 / \mathrm{rbmfc} 9(31) 684$.

5. Pesquisa de Orçamentos Familiares - 2008-2009: Antropometria e estado nutricional de crianças, adolescentes e adultos no Brasil. Rio de Janeiro. Instituto Brasileiro de Geografia e Estatística (IBGE). 2010. Disponível em http:// www.ibge.gov.br/home/. [Acesso em 11 Jan 2015].

6. Instituto Nacional de Alimentação e Nutrição (INAN). Pesquisa Nacional sobre Saúde e Nutrição (PNSN- 1989). Brasília: Ministério da Saúde; 1989. Disponível em: http:// dab.saude.gov.br/portaldab/ape _ vigilancia_alimentar. php?conteudo = pnsn. [Acesso em 11 Jan 2015].

7. Pazin JR, Donadone VS, Abreu ES, Simony RF. Prevalência de sobrepeso e obesidade em pré-escolares e escolares de escolas particulares. Rev Ciên Saúde 2012;5(2):87-91.

8. Granville-Garcia AF, Menezes VA, Lira PIC, Serqueira LA, Ferreira JMS, Cavalcanti AL. Prevalência de sobrepeso e obesidade em pré-escolares de escolas públicas e privadas em Recife, Pernambuco, Brasil. Cad Saúde Colet 2009; 17(4):989-1000.

9. Petroski EL. Antropometria: Técnicas e Padronizações. $5^{\circ}$ Ed. Fontoura: Várzea Paulista - SP; 2011.

10. Center for Disease Control and Prevention. Body Mass Index: Considerations for Practitioners. Disponível em: http://www. cdc.gov/obesity/downloads/BMlforPactitioners.pdf. Acesso em: 27 de outubro de 2015.

11. Souza WC, Souza WB, Grzelczak MT, Mascarenhas LPG, Lima VA. Comparação de IMC em meninos e meninas de 5 e 6 anos de idade de uma escola do município de Três BarrasSC. Rev Uniandrade 2013;14(2):121-30.
12. Hoehr CF, Reuter CP, Tornquist L, Nunes HMB, Burgos MS. Prevalence of obesity and hypertension in schoolchildren: comparative study between rural schools in the municipality of Santa Cruz do Sul/RS. Rev Epidemiol Control Infec 2014;4(2):122-6. DOI: 10.17058/reci.v4i24772.

13. Souza WC, Mascarenhas LPG, Reiser FC, Souza WB, Grzelczak MT. Estudo comparativo da prevalência de sobrepeso e obesidade em pré-escolares de Três Barras SC. Saúde e Meio Ambiente 2014;3(1):114-20.

14. Gigante DP, Victora CG, Araújo CLP, Barros FC. Tendências no perfil nutricional das crianças nascidas em 1993 em Pelotas, Rio Grande do Sul, Brasil: análises longitudinais. Cad Saúde Pública 2003;19(Supl 1):141-7. DOI: 10.1590/S0102311X2003000700015.

15. Colloca EA, Duarte ACGO. Obesidade infantil: etiologia e encaminhamentos, uma busca na literatura. In: II Seminário de Estudos em Educação Física Escolar; 2008, São Carlos. Anais... São Carlos: CEEFE/UFSCar 2008;189-221.

16. Costa RF, Cintra IP, Fisberg M. Prevalência de sobrepeso e obesidade em escolares da cidade de Santos - SP. Arq Bras Endocrinol Metab 2006;50(1):60-7. DOI: 10.1590/S000427302006000100009.

17. Ronque ERV, Cyrino ES, Dórea VR, Serassuelo Júnior $H$, Galdi EHG, Arruda M. Prevalência de sobrepeso e obesidade em escolares de alto nível socioeconômico em Londrina, Paraná, Brasil. Rev Nutr 2005;8(6):709-17. DOI: 10.1590/ S1415-52732005000600001.

18. Paula FAR, Lamboglia CMGF, Silva VTBL, Monteiro MS, Moreira AP, Pereira MHN, et al. Overweight and obesity prevalence in students from public and private system in the city of Fortaleza. Rev Bras Promoc Saúde 2014;27(4):45561. DOI: $10.5020 / 18061230.2014 . p 455$.

19. Rosaneli $C F$, Auler F, Manfrinato $C B$, Rosaneli $C F$, Sganzerla C, Bonatto MG, et al. Avaliação da prevalência e de determinantes nutricionais e sociais do excesso de peso em uma população de escolares: análise transversal em 5.037 crianças. Rev Assoc Med Bras 2012;58(4):472-6. DOI: 10.1590/S0104-42302012000400019.

Como citar: SOUZA, William Cordeiro de; LIMA, Valderi Abreu de; MASCARENHAS, Luis Paulo Gomes. Frequência de excesso de peso em pré-escolares. Cinergis, Santa Cruz do Sul, v. 17, n. 1, mar. 2016. ISSN 2177-4005. Disponível em: <https:// online.unisc.br/seer/index.php/cinergis/article/view/6825>. Acesso em: . doi:http://dx.doi.org/10.17058/cinergis.v17i1.6825. 